\title{
Production of Secondary Cosmic Rays in Supernova Remnants
}

\section{E. G. Berezhko, L. T. Ksenofontov*}

Yu. G. Shafer Institute of Cosmophysical Research and Aeronomy, 31 Lenin Ave., 677891

Yakutsk, Russia

E-mail: ksenofoneikfia.sbras.ru

\begin{abstract}
We study production of secondary cosmic rays (CR) in supernova remnants (SNR). The model includes reacceleration of already existing in interstellar medium secondary CR particles as well as creation of secondary CRs in nuclear collisions of accelerated protons with gas nuclei and their subsequent acceleration by SNR shock. It is shown that production secondary CRs in SNRs produces considerable effect in their resultant energy spectrum making it essentially flatter above $10 \mathrm{GeV}$. It is shown that calculated energy spectra of antiprotons, positrons and secondary nuclei $\mathrm{Li}, \mathrm{Be}, \mathrm{B}$ are well consistent experimental data obtained in recent experiments PAMELA, Fermi and AMS-02.
\end{abstract}

The 34th International Cosmic Ray Conference,

30 July- 6 August, 2015

The Hague, The Netherlands

* Speaker. 


\section{Introduction}

There is a great astrophysical interest in cosmic ray (CR) positrons. It is believed that most of positrons originate in collisions of CR protons with interstellar medium (ISM) gas nuclei. Therefore positrons represent a kind of so-called secondary CR component, opposite to the primary CRs, which originate in CR sources, presumably in supernova remnants (SNRs). The same is trough for antiprotons, which are the other kind of secondary CR component.

The positron energy spectrum measured recently in PAMELA, Fermi and AMS-02 experiments at kinetic energy $\varepsilon>10 \mathrm{GeV}$ turned out to be much flatter than it is expected for positrons created in p-p collisions in ISM. This stimulated many kind of assumptions that significant part of positrons originate from new astrophysical sources such as pulsars or the annihilation of dark matter particles (see [23] for a review).

At the same time SNRs are not only the most probable sources of primary CRs with energies below $10^{17} \mathrm{eV}$ [14], but also contribute significantly in the production of secondary CRs due to reacceleration of already existing in ISM CRs and due to nuclear collisions of primary CR particles with gas nuclei leading to the creation of secondary particles which undergo subsequent acceleration by SNR shock. The detailed study of these processes for the case of secondary nuclei [13] and antiprotons [12] based on the nonlinear kinetic theory of CR acceleration in SNRs [10] demonstrated that SNRs are expected to contribute significantly to secondary CR spectra at kinetic energies $\varepsilon>10 \mathrm{GeV} /$ nucleon making it significantly flatter. Therefore it is natural to suggest that the observed flattering of positron energy spectrum at $\varepsilon>10 \mathrm{GeV}$ can be partly or even predominantly due to SNR contribution. Simple estimate [15] and the preliminary study [11,8] have indeed confirmed that the observed flat high energy positron spectrum is consistent with the expected SNR contribution.

Here we calculate the energy spectrum of positrons produced in SNRs to compare it with the existing data and make the prediction at higher energies $\varepsilon>400 \mathrm{GeV}$ where experimental data are not existed yet.

\section{Production of secondary CRs in SNRs}

Injection of secondary CRs (and primary CRs as well) into the shock acceleration process in SNRs was described in details in our previous publications $[13,12]$. Therefore we briefly mention here the most relevant aspects of this process.

Some small fraction of the postshock thermal gas particle population are able to recross the shock that means the beginning of their shock acceleration. This is the most general and the most intense injection mechanism. It occurs for all kind of ions and electrons existing in the ISM and therefore it is relevant for primary CRs only. The corresponding injection rate is determined by the number of particles involved into the acceleration from each medium volume crossed the shock. In the case of nuclei it is described by the dimensionless injection parameter $\eta \sim 10^{-3}$. There is a good semi-quantitative understanding of the injection rate of nuclear particles into the diffusive shock acceleration process in terms of the escape of suprathermal downstream particles. However, due to the incompletely known relaxation state of the downstream ions, the escape rate is theoretically known only up to factors of order unity, and therefore it is necessary to fix its value empirically in 
quantitative model for the SNR as a whole. The actual value of $\eta$ can be extracted from the fit of the observed SNR synchrotron spectrum, which shape is sensitive to the shock modification due to the CR backreaction, that in turn strongly depends on $\eta$. Detailed considerations show that for all known young Galactic SNRs the properties of the observed nonthermal emission are consistent with the injection rate within the range $\eta=10^{-4} \div 10^{-3}[9,8]$.

The content of secondary CRs like secondary nuclei $\mathrm{Li}, \mathrm{Be}, \mathrm{B}$ or positrons and antiprotons in ISM is so small that they can not be produced due to such an injection in any appreciable amount.

The electron injection mechanism is very poorly known compared with the injection of protons. Since at relativistic energies they have exactly the same dynamics as the protons their distribution function at any given time can be represented in the form $f_{\mathrm{e}}(p)=K_{\mathrm{ep}} f_{\mathrm{p}}(p)$ in terms of electron-to-proton ratio $K_{\mathrm{ep}} \ll 1$ for all the momenta $p$ exceeding the injection momentum of protons. The electron distribution function $f_{\mathrm{e}}(p)$ deviates from this relation only at sufficiently large momenta $p$ due to synchrotron losses. The parameter value $K_{\text {ep }}$ at a given proton injection rate $\eta$ determines the injection rate of electrons.

Kinetic energy of all kind of Galactic cosmic ray (GCR) particles is considerably larger then the energy of gas particles injected from the postshock thermal pool. Therefore all GCRs which meet the expanding SNR shock are naturally involved into the diffusive shock acceleration. CR acceleration due to this second relevant injection mechanism is usually called "reacceleration". This term is used to distinguish the process of further increase of energy of already energetic particles due to interactions with SNR shocks during their propagation in ISM. In this regard it is similar to the stochastic acceleration (also called reacceleration) of GCRs due to their interactions with the background MHD-turbulence.

Since GCR energy spectra are relatively steep and it have a peak at kinetic energy $\varepsilon=\varepsilon_{\mathrm{GCR}} \sim$ $1 \mathrm{GeV}$ their injection parameters (the number density and mean momentum of the injected particles) can be represented in the form $N_{\mathrm{inj}}=N_{\mathrm{GCR}}, p_{\mathrm{inj}}=p_{\mathrm{GCR}}$, where $N_{\mathrm{GCR}}$ is the total number of GCR species per unit volume and $p_{\mathrm{GCR}}$ is their mean momentum, that corresponds to $\varepsilon_{\mathrm{GCR}}$.

In order to determine the number of reaccelerated CRs we starts from the case of protons. Integration of the protons spectrum at energies above $0.3 \mathrm{GeV}$ gives the number density of $\mathrm{CR}$ protons $N_{\mathrm{GCR}}=3.3 \times 10^{-10} \mathrm{~cm}^{-3}$.

Since at $\mathrm{GeV}$ energies the flux of $\mathrm{CR}$ electrons is by a factor of 100 lower then the protons and the flux of positrons is about 0.2 of electron flux the values of number density of CR electrons and positrons in ISM are $N_{\mathrm{GCR}}=3.3 \times 10^{-12} \mathrm{~cm}^{-3}$ and $N_{\mathrm{GCR}}=6.6 \times 10^{-13} \mathrm{~cm}^{-3}$ respectively.

Primary nuclei (mainly protons) during their acceleration inside SNRs produce secondary CRs in nuclear collisions with the background gas like GCRs do it in the Galactic disk. Essential fraction of these already energetic particles has possibility to be involved in further shock acceleration. This is the third mechanism of secondary CR production inside SNRs. For the first time it was studied to describe the formation of the secondary CR nuclei spectra [13].

The production rate of secondary CR particles due to the nuclear collisions of primary CRs with the gas nuclei within SNR can be described by the source term

$$
q_{\mathrm{s}}(r, p, t)=4 \pi c N_{\mathrm{g}} \int_{0}^{\infty} d p^{\prime} p^{\prime 2} \sigma_{\mathrm{ps}}\left(p, p^{\prime}\right) f_{\mathrm{p}}\left(r, p^{\prime}, t\right),
$$

in the transport equation for the distribution function of secondary CRs $f_{\mathrm{s}}(r, p, t)$. Here $\sigma_{\mathrm{ps}}\left(p, p^{\prime}\right)$ is 
inelastic cross-section of secondary CRs production with momentum $p$ in the collision of primary (parent) nuclei of momentum $p^{\prime}$ with the gas nuclei, $t$ is the time interval since the supernova explosion, $r$ is the radial distance from the presupernova star. In the case of positrons all kind of accelerated in SNRs nuclei (predominantly protons) play a role of parent nuclei. We use analytical expressions for the inelastic cross-section of proton-proton interaction leading to the creation of electrons and positrons $\sigma_{\mathrm{ps}}\left(p, p^{\prime}\right)$ derived in [19]. Reacceleration and the acceleration of nuclei created in SNRs in nuclear collisions are of the prime importance for the secondary CRs even though relatively small part of primary CRs is also produced due to these processes.

The source term $q_{\mathrm{s}}$ describes the creation of secondaries throughout the remnant, everywhere downstream and upstream of SNR shock up to the distances $d \sim l_{\mathrm{p}}\left(p^{\prime}\right)$ of the order of the diffusive length $l_{\mathrm{p}}\left(p^{\prime}\right)$ of their parent primary CRs. Essential part of these particles are naturally involving in the acceleration at SNR shock. It includes all the particles created upstream and the particles created downstream at distances less then their diffusive length $l_{\mathrm{s}}(p)$ from the shock front. The number of these particles is increasing function of their momentum because $l \propto \kappa(p) \propto p$ for the Bohm type diffusion coefficient $\kappa(p)$ which is realized during efficient CR acceleration in SNRs [9]. This makes the secondary particle spectra

$$
N_{\mathrm{s}}(p, t)=16 \pi^{2} p^{2} \int_{0}^{\infty} d r r^{2} f_{\mathrm{s}}(r, p, t),
$$

produced in SNR at any given evolutionary epoch $t$, harder compared with the spectra of primaries $N_{\mathrm{p}}(p, t)$.

The SNR efficiently accelerates CRs up to some maximal age $T_{\mathrm{SN}}$ when SNR release all previously accelerated CRs, primaries and secondaries, with the spectra $N_{\mathrm{p}}(\varepsilon)=v^{-1} N_{\mathrm{p}}\left(p, T_{\mathrm{SN}}\right)$ and $N_{\mathrm{S}}(\varepsilon)=v^{-1} N_{\mathrm{s}}\left(p, T_{\mathrm{SN}}\right)$ respectively, into surrounding ISM. Here $\varepsilon$ and $v$ are the kinetic energy and speed of particle with momentum $p$.

These CRs released from SNRs together with secondary CRs produced in ISM form the total secondary $n_{\mathrm{s}}(\varepsilon)$ CR populations

$$
n_{\mathrm{s}}=n_{\mathrm{s}}^{\prime}+n_{\mathrm{s}}^{\prime \prime}
$$

where $n_{\mathrm{s}}^{\prime}(\varepsilon)$ represents the energy spectrum of secondaries produced in nuclear collisions of primary CRs within the Galactic disk and $n_{\mathrm{s}}^{\prime \prime}(\varepsilon)$ is the average energy spectrum of secondaries in the Galactic disk volume produced in SNRs and modified due to their propagation effect. We restrict the analysis to CR electrons and positrons not affected by the solar modulation, the ones with energies $\varepsilon>10 \mathrm{GeV}$, for which the effects of their escape from the Galaxy can be neglected. Within the leaky box model the energy spectrum of secondaries modified due to their energy losses in ISM after their release from the sources is given by the expression [24]

$$
n_{\mathrm{S}}^{\prime \prime}(\varepsilon)=\frac{v_{\mathrm{SN}} \tau_{\mathrm{loss}}(\varepsilon)}{V_{\mathrm{G}} \varepsilon} \int_{\varepsilon}^{\infty} d \varepsilon^{\prime} N_{\mathrm{s}}\left(\varepsilon^{\prime}\right)
$$

where $N_{\mathrm{S}}(\varepsilon)$ is the overall spectrum of electrons or positrons released from a single SNR, $v_{\mathrm{SN}}$ is the supernova explosion rate, $V_{\mathrm{G}}$ is the CR confinement volume, $\tau_{\text {loss }}(\varepsilon)$ is the loss time determined by inverse Compton scattering and synchrotron emission. For $\tau_{\text {loss }}(\varepsilon)$ we use the calculation [24] which corresponds to the relevant photon fields: cosmic microwave background, the Galactic starlight and far-infrared photons from the dust emission with energy density $0.3 \mathrm{eV} \mathrm{cm}^{-3}$. 
For the energy spectrum of electrons and positrons produced in $\operatorname{ISM~} n_{\mathrm{s}}^{\prime}(\varepsilon)$ we use the calculation performed by [21] within their GALPROP model.

\section{Results and discussion}

We have calculated the overall energy spectra of all relevant CR species accelerated in SNRs, within kinetic nonlinear model. The model is based on a fully time-dependent self-consistent solution of the CR transport equation together with the gas dynamic equations in spherical symmetry. It includes the most relevant physical factors, essential for the evolution and CR acceleration in a SNR and it is able to make quantitative predictions of the expected properties of CRs produced in SNRs and their nonthermal radiation. The application of the theory to individual SNRs has demonstrated its capability of explaining the observed SNR nonthermal emission properties $[9,8]$. The theory is able to explain major characteristics of the observed CR spectrum up to an energy of $\sim 10^{17} \mathrm{eV}[10,9]$. Similar approach was developed recently by other authors $[22,18]$.

We restrict ourselves by the most simple case of type Ia SN in uniform ISM with corresponding SN parameter values: explosion energy $E_{\mathrm{SN}}=10^{51} \mathrm{erg}$ and ejecta mass $M_{\mathrm{ej}}=1.4 M_{\odot}$. We use typical values of the dimensionless parameter $\eta=3 \times 10^{-4}$, which describes the injection of gas particles into the shock acceleration [25]. We consider the typical ISM with hydrogen number density $N_{\mathrm{H}}=1.5 \mathrm{~cm}^{-3}$ and magnetic field values $5 \mu \mathrm{G}$, which roughly corresponds to the average ISM within the Galactic disk. We adopt time-independent upstream magnetic field value $B_{0}$ and ignore magnetic field amplification effect because the secondaries are mainly produced at the late evolutionary phases [13] when this effect becomes irrelevant.

We perform selfconsistent calculation up to the SNR age $T_{\mathrm{SN}}$ when SNR release all previously accelerated CRs into surrounding ISM. As it was pointed out [13] there are at least two conditions which determine the confinement time $T_{\mathrm{SN}}$. The first one is the decrease of the shock Mach number during the SNR evolution. When it becomes so low, at some stage $t=t_{4}$, such that the shock compression ratio $\sigma$ drops below 4 , the acceleration of freshly injected particles becomes inefficient. The acceleration process may also stop at some stage $t=t_{6}$ when the postshock temperature drops below $10^{6} \mathrm{~K}$, because radiative SNR cooling sets in strongly. Since the SNR looses a large amount of its energy, efficient CR acceleration terminates presumably at this stage. For the considered case of ISM with gas number $N_{\mathrm{H}}=1.5 \mathrm{~cm}^{-3}$ these two physical factors come into play at about the same time of $10^{5} \mathrm{yr}$ [13]. Therefore we adopt the value $T_{\mathrm{SN}}=10^{5} \mathrm{yr}$ appropriate for considered ISM density.

GCRs at kinetic energy $\varepsilon \approx 1 \mathrm{GeV}$ are characterized by the electron to proton ratio $K_{\mathrm{ep}}=10^{-2}$. Since the main part of CRs produced in SNRs are released in the surrounding ISM at late Sedov SNR evolutionary phase the value $K_{\mathrm{ep}}=10^{-2}$ characterizes the injection of protons and electrons at this late stage. At the same time for all known young SNRs the value of this parameter extracted from the fit of the SNRs nonthermal emission properties is considerably lower: $K_{\text {ep }} \sim 10^{-4}$ (e.g. [9]). This situation suggests the increase of electron injection rate during SNR evolution so that electron to proton ratio $K_{\mathrm{ep}}(t)$ is increasing function of SNR evolutionary time $t$. We use in our calculations $K_{\mathrm{ep}}=10^{-4}$ at $t<10^{4} \mathrm{yr}$ and $K_{\mathrm{ep}}=10^{-4}\left(t / 10^{4} \mathrm{yr}\right)^{2}$ at $10^{4}<t<10^{5} \mathrm{yr}$.

Calculated intensities of electrons end positrons as a function of energy are presented in Fig.1 together with the existing data. For the energy spectra of electrons $n_{\mathrm{e}}^{\prime}(\varepsilon)$ and positrons $n_{\mathrm{p}}^{\prime}(\varepsilon)$ we 


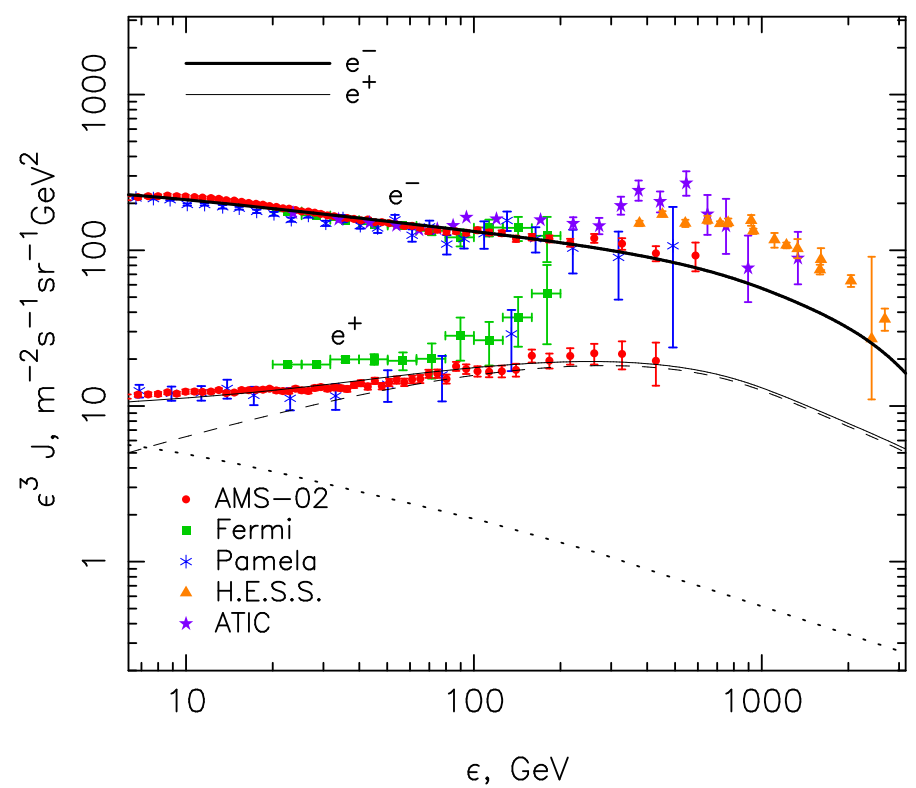

Figure 1: Calculated electron (thick line) and positron (thin lines) spectra produced inside SNRs and in ISM together with PAMELA [4, 5], Fermi LAT [2], HESS [7], ATIC [16] and AMS-02 [6] data. Dotted and dashed lines represent spectra of positrons created in p-p collisions in ISM [21] and inside SNR respectively.

use the results of calculation performed by [21].

It is seen that calculated positron spectrum is considerably flatter then the electron spectrum due to the considerable SNRs contribution. At energies from 10 to $400 \mathrm{GeV}$ the calculated spectra are well consistent with the recent PAMELA and AMS-02 data, appeared after our preliminary publication [11].

Note that the amount of positrons produced in SNRs according to our calculation at energies $\varepsilon=0.1-1 \mathrm{TeV}$ is considerably lower then earlier estimate [15]. This is presumably due to the number of simplification used in this estimate.

Relative contribution of two different mechanisms of secondary CR injection into the shock acceleration in SNRs is determined by the ISM gas number density $N_{\mathrm{H}}$. Reacceleration of GCRs dominates in the case of SNR expanding into the diluted ISM with $N_{\mathrm{H}} \ll 1 \mathrm{~cm}^{-3}$ whereas the acceleration of secondaries created in SNR in nuclear collisions is dominant at high density ISM with $N_{\mathrm{H}} \gtrsim 0.1 \mathrm{~cm}^{-3}$ [13]. In the considered case of typical ISM number density $N_{\mathrm{H}}=1.5 \mathrm{~cm}^{-3}$ reacceleration of GCR positrons contributes about $10 \%$ in their resultant spectrum.

The positron to positron plus electron ratio as a function of energy is represented in Fig.2. According to our calculation the increase of the ratio with energy above $10 \mathrm{GeV}$ is due to SNRs contribution into the positrons production. It is also seen that calculated ratio agrees well with PAMELA and AMS-02 data.

SNR contribution into the secondary CR spectra represents the component which is unavoidably expected if SNRs are the main source of GCRs. Comparison of calculated energy spectra of electrons and positrons with the existing data leads us to a conclusion that the observed high energy flat positron spectrum is predominantly produced in Galactic SNRs. This enable to expect similar excess in the antiproton energy spectrum. The existing PAMELA data, well agrees with 


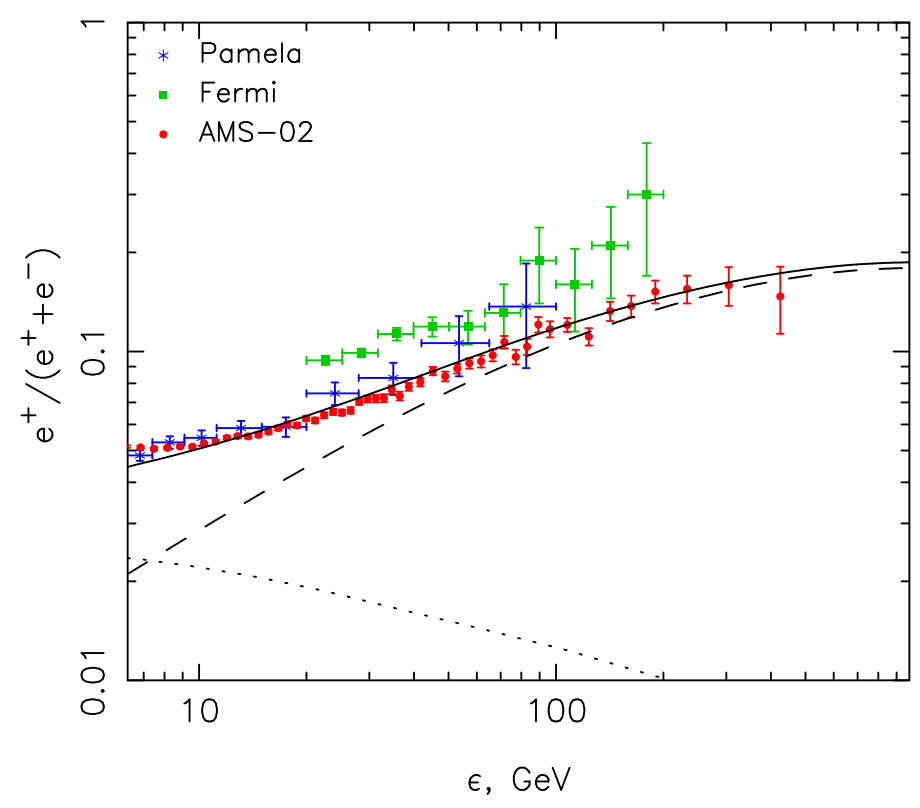

Figure 2: Calculated positron to electron plus positron ratio as a function of kinetic energy together with the results of PAMELA [5], Fermi [2] and AMS-02 [1] experiments. The dashed (dotted) line represents the ratio, which includes positrons produced in SNRs (ISM) only; the solid line corresponds to the sum of these positron components.

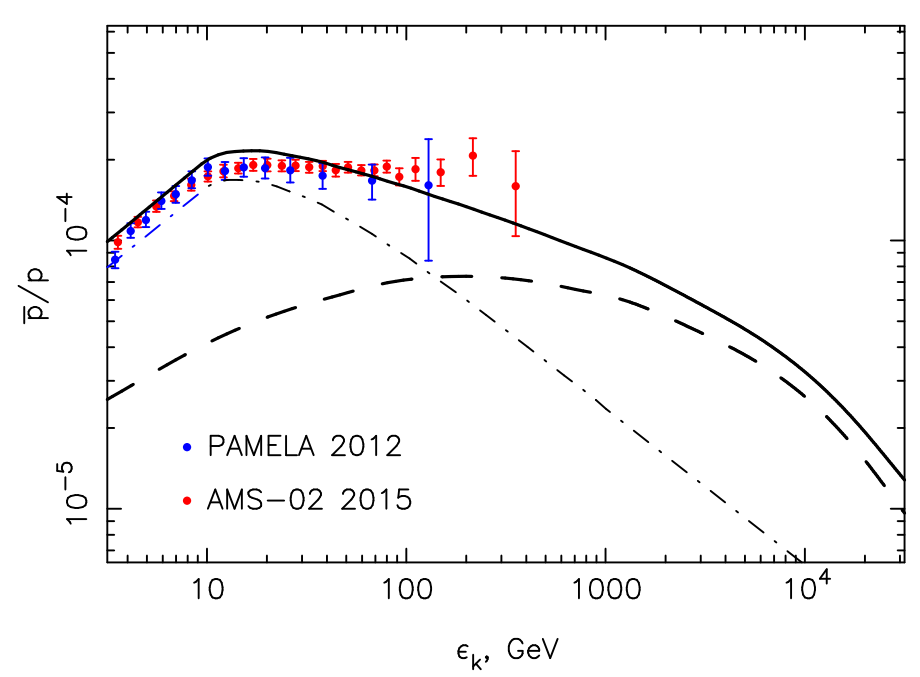

Figure 3: Calculated antiproton-to-proton ratio as a function of energy together with PAMELA [3] and preliminary AMS-02 [20] data. Dash-dotted line corresponds to the spectrum of antiprotons created in p-p collisions in ISM [17], dashed line corresponds to created in p-p collisions inside SNRs plus reaccelerated in SNRs, solid line represent the sum of contributions of these processes. 
our calculation within the energy range $10 \mathrm{GeV}<\varepsilon<100 \mathrm{GeV}$ and provide the evidence that the actual ratio $\bar{p} / p$ is indeed more flat than it is expected if antiprotons are created in ISM only [12]. The new data from AMS-02 experiment [20] presented in Fig.3 made it clear that the actual ratio $\bar{p} / p$ is indeed dominated by the SNRs contribution at energies $\varepsilon>10 \mathrm{GeV}$.

This work is supported by the Russian Foundation for Basic Research (grants 13-02-00943 and 13-02-12036) and by the Council of the President of the Russian Federation for Support of Young Scientists and Leading Scientific Schools (project No. NSh-3269.2014.2).

\section{References}

[1] L. Accardo, et al., Phys. Rev. Lett. 113 (2014) 121101.

[2] M. Ackermann, et al., Phys. Rev. Lett. 108 (2012) 011103.

[3] O. Adriani, et al., Phys. Rev. Lett. 105 (2010) 121101.

[4] O. Adriani, et al., Phys. Rev. Lett. 106 (2011) 201101.

[5] O. Adriani, et al., Phys. Rev. Lett. 111 (2013) 081102.

[6] M. Aguilar, et al., Phys. Rev. Lett. 113 (2014) 121102.

[7] F. Aharonian, et al., Phys. Rev. Lett. 101 (2008) 261104.

[8] E.G. Berezhko, Nuclear Phys. B (Proc. Suppl.) 256 (2014) 23.

[9] E.G. Berezhko, Adv. Space Res. 41 (2008) 429.

[10] E.G. Berezhko, V.K. Elshin \& L.T. Ksenofontov, J. Exp. Theor. Phys. 82 (1996) 1.

[11] E.G. Berezhko \& L.T. Ksenofontov, J. Phys.: Conf. Ser. 409 (2013) 012025.

[12] E.G. Berezhko \& L.T. Ksenofontov, Astrophys. J. 791 (2014) L22.

[13] E.G. Berezhko, L.T. Ksenofontov, V.S. Ptuskin, H.J. Völk \& V.N. Zirakashvili, Astron. Astrophys. 410 (2003) 189.

[14] E.G. Berezhko, \& H.J. Völk, Astrophys. J. 661 (2007) L175.

[15] P. Blasi, Phys. Rev. Lett. 103 (2009) 051104.

[16] J. Chang, et al., Nature 456 (2008) 362.

[17] F. Donato, et al., Astrophys. J. 563 (2001) 172.

[18] H. Kang, J. Korean Astron. Soc. 43 (2010) 25.

[19] S.R. Kelner, F.A. Aharonian \& V.V. Bugayov, Phys. Rev. D 74 (2006) 034018.

[20] A. Kounine, Talk at the "AMS Days at CERN" (2015) http://indico.cern.ch/ event/381134/.

[21] I.V. Moskalenko \& A.W. Strong, Astrophys. J. 493 (1998) 694.

[22] V.S. Ptuskin, V.N. Zirakashvili \& E.-S. Seo, Astrophys. J. 718 (2010) 31.

[23] P.D. Serpico, Astropart. Phys. 39 (2012) 2.

[24] L. Stawarz, V. Petrosian \& R. Blandford,, Astrophys. J. 710 (2010) 236.

[25] H.J. Völk, E.G. Berezhko, \& L.T. Ksenofontov, Astron. Astrophys. 409 (2003) 563. 